\title{
EL INGENIERO Y LA SEGURIDAD: LA SEGURIDAD INTEGRADA
}

\author{
(THE ENGINEER AND SAFETY: INTEGRATED SAFETY)
}

José Gascón y Marín Pérez, Ingeniero de Caminos, Canales y Puertos ESPAÑA

\section{RESUMEN}

Trabajo que nos presenta el hecho de que la Prevención es algo que ya intervenía y actualmente interviene en el Proceso Productivo de la Construcción, todo ello desde la óptica de la ingeniería y de la Obra Civil.

\section{SUMMARY}

This paper evidences the fact that Prevention has been and currently is a part of the Construction Production Process from the point of view of engineering and Civil Works.
Parece que en los últimos tiempos la seguridad se ha puesto de moda: campañas de prensa y televisión, noticias, anuncios por la calle, proliferación de cursillos, jornadas, congresos, etc.

El problema, sin embargo, no es tan nuevo, puesto que siempre que el hombre ha realizado diversos trabajos de construcción, fabricación, etc., ha sufrido por desgracia el accidente de trabajo, y, a lo largo del tiempo, se le ha ido prestando atención.

Nos lo demuestra, sin salirnos de nuestro país, que ya en las Leyes de Indias se dieron normas sobre los trabajos de los indios en las mismas; que en la construcción del Monasterio de El Escorial había una especie de Hospital para los canteros que allí trabajaban. En 1778 el Consejo de Castilla dio un edicto hablando de la peligrosidad de las obras y obligaciones de los Aparejadores, y que era un tema con impacto nos lo demuestra también, más recientemente, el conocido tapiz de Goya del albañil accidentado, y el cuadro de Sorolla.

Sin embargo, no se puede negar que en los últimos tiempos ha habido una más clara toma de conciencia sobre el problema de los accidentes en el trabajo y de cómo plantear la lucha contra ellos, y mejor aún, la prevención, toma de conciencia que podemos decir que simultáneamente se ha hecho realidad por los organismos correspondientes de la Administración y por las Industrias a través de sus Empresas.

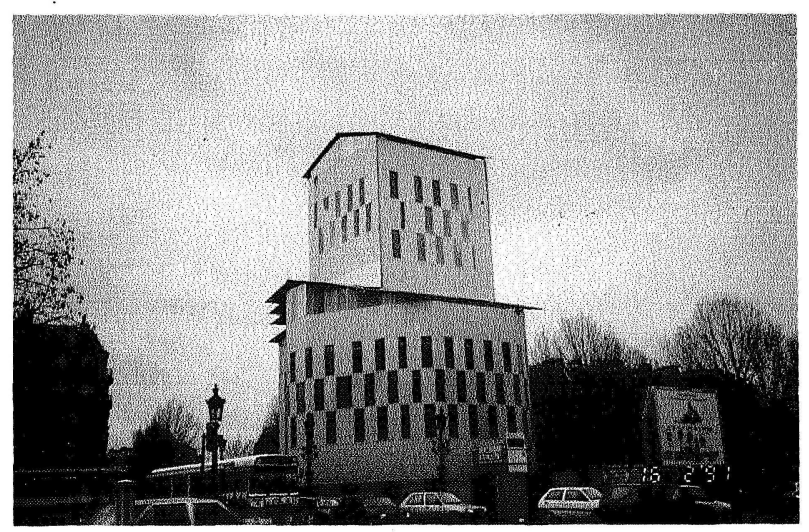

La inseguridad en lo provisional. 


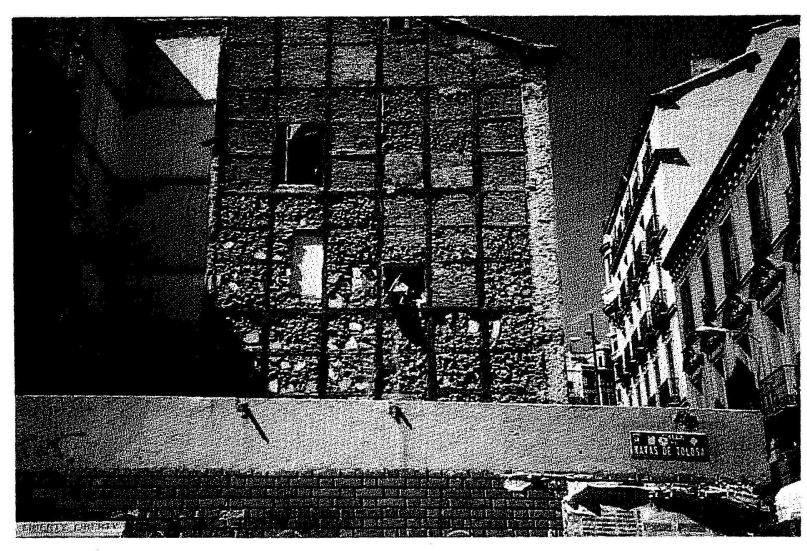

La edificación antigua como víctima de la falta de medidas de seguridad.

Todo ello tal vez sea el resultado de ver cómo los dos índices que se manejan más frecuentemente para valorar la importancia de estos accidentes, el de Frecuencia y el de Gravedad, suben de forma más que alarmante, en general.

A título de ejemplo citaremos que entre los años $50 \mathrm{y}$ 60 el número de accidentes ocurridos en España se duplicó y, en órden de magnitud, pasa de 500.000 a 1.000 .000 , cifra que en años posteriores se mantuvo sensiblemente pero contrapesada, por desgracia, con la importancia de los mismos, ya que el número de accidentes mortales se duplicó en estos años en que el número se mantenía sensiblemente constante.

Lo primero que tendríamos que plantearnos es qué entendemos por accidente, y con la Legislación hoy día vigente en España se toma como accidente "toda lesión corporal que el trabajador sufra con ocasión o por consecuencia del trabajo que ejecute por cuenta ajena", con lo que queda claro que cuenta el número de accidentes por las personas afectadas cuando se produce el mismo y no únicamente la causa que ha producido éste, que puede dar lugar a varias personas lesionadas simultáneamente.

Indudablemente, todo planteamiento para luchar contra los accidentes, y por lo tanto trabajar en seguridad, debe estar basado, en primer lugar y fundamentalmente, en el aspecto humano que encierra por el sufrimiento del propio accidentado, cuya gravedad puede llegar hasta la muerte; y las repercusiones, también humanas, que en sus familiares, amigos, y en la misma Empresa, se producen por causa del accidente, así como en la sociedad.

Éste debe ser, pues, nuestro motor fundamental para trabajar en este campo con gran vocación y mejorando nuestros conocimientos para una mejor lucha contra el accidente. Pero hay un segundo aspecto, que es la consecuencia económica del accidente en el propio accidentado, en su familia, en la Empresa, y, en definitiva, en la Sociedad. Recordemos que cuando últimamente se ha tratado en Conferencias y en la Prensa de este asunto, se han manejado siempre cifras de varios miles de millones de pesetas que cuesta al conjunto del país anualmente los accidentes.

$\mathrm{Al}$ accidentado y su familia le afecta porque a pesar de la Seguridad Social (que en estos últimos años ha mejorado sus prestaciones) con frecuencia ven mermados algunos de los conceptos, como primas, gratificaciones, etc.

A la Empresa, por lo que supone a la misma desde el punto de vista de seguros y accidentes y las pérdidas que en su cadena de producción tiene luego al producirse un accidente.

Y, en definitiva, al país, por lo que de forma directa representa en horas perdidas de trabajo y en capital para pensiones con que hacer frente a incapacidades y muertes.

No olvidemos que, cuando se habla de estos costos, la mayor parte de las veces se está valorando únicamente el costo directo que, aun dentro de algunas dificultades, es fácil de calcular, pero que el total de los gastos ocasionados por los accidentes sería aún muchísimo mayor si llegaramos a cifrar su total repercusión económica, ya que los peritos en la materia nos dicen que este coste indirecto oscila de 2 a 4 veces el costo directo.

Por último, si estas razones, humanitarias y cristianas en primer lugar, y económicas después, no fueran bastantes, no podemos olvidar las posibles responsabilidades en que se puede incurrir en relación con los accidentes. Hoy ante el juez no se supone como antes que era imprudencia, y éste tenía que demostrar que era delito, sino que supone que lo es y el acusado tiene que defenderse.

Por otra parte, al plantearnos el tema de la Seguridad desde el plano que más nos afecta, o sea el de la indus- 
tria de la Construcción en general, en su doble faceta de lo que se viene llamando Obra Civil y Edificación, tampoco podemos olvidar desde el principio del planteamiento las características específicas que tiene esta actividad nuestra (al decir nuestra estoy pensando en la vida profesional de la gran mayoría de los Ingenieros de Caminos, que bien desde la Administración o en la empresa privada, tanto de proyecto como de explotación y construcción, se relacionan con la construcción de obras muy diversas por su volumen, por su localización, o por su técnica, etc., pero, en definitiva, Industria de la Construcción).

Por desgracia, el trabajo en la Industria de la Construcción encierra muchos riesgos. Ello hace que esta actividad industrial sea altamente peligrosa, siendo por lo tanto necesaria, importante y urgente una labor de prevención de accidentes dentro de su ámbito, ya que, después de la Industria Minera, alcanza los mayores índices de la totalidad de la Industria.

Entre las causas de esta gran peligrosidad que diferencia a la Industria de la Construcción de las demás vamos a citar algunas:

1. Cambio continuo del emplazamiento en los centros de trabajo (Obras). Los plazos de permanencia de estos centros son cada vez más reducidos debido a que los tịempos de ejecución son cada vez más cortos.

Es mucho más difícil planificar la seguridad de una obra que tiene un período de vida breve, que en una industria fabril, con emplazamiento fijo durante muchos años y con una actividad normalmente poco cambiante.

2. Trabajo variado y cambiante. En un reducido plazo, el de ejecución de la obra, se han de hacer trabajos variadísimos, que van desde excavar unos cimientos, hasta impermeabilizar una cubierta, pasando por preparación y colocación de hierro y de encofrados, vertido de hormigón, trabajos de electricidad, fontanería, etc., o de explotación de una cantera o puesta en obra de grandes masas de hormigón (en una presa), explosivos y perforaciones (túneles).
3. Otro condicionamiento de alta accidentalidad de la Industria de la Construcción es el continuo trasiego de la mano de obra. Muchos hombres sólo usan la Industria de la Construcción como etapa de tránsito en su vida laboral, o trabajo, puente entre su procedencia, en general de la agricultura, y su meta final, que suele ser conseguir un puesto fijo en otras industrias más estables o en los servicios, y también el bajo nivel cultural.

4. También podemos citar el medio ambiente en que se desarrolla el trabajo, casi siempre al aire libre y expuestos, por lo tanto, a las inclemencias del tiempo. En emplazamientos frecuentemente alejados de centros urbanos, con todas las secuelas que esta circunstancia trae consigo, tales como largos desplazamientos, dificultad de asistencia adecuada e inmediata en caso de accidente, etc.

5. Asimismo, hay que señalar la continua evolución de los medios de trabajo, con empleo de mecanismo cada vez más variado, teniendo sin embargo como contrapartida la baja calificación técnica que en general tienen los trabajadores de la Construcción y que no deja de ser motivo creciente de peligrosidad al manejar los citados medios mecánicos. Si bien hoy día hay técnicas nuevas que, por el contrario, alejan el posible accidente (hinca de tubos en horizontal).

6. ${ }^{a} \quad$ El boom en la Construcción, con la fecha de 1992 (Barcelona, Sevilla y Madrid), con plazos fijos.

Entre las primeras podemos citar las instalaciones en sí, la maquinaria, los procesos de fabricación, la organización del trabajo y la capacitación profesional.

En las psicofisiológicas, unas que podríamos llamar médicas, patológicas y estado psíquico del accidentado, la falta de atención, la fatiga, la irresolución, etc.

Se estima que en muchos casos se mejora por las máquinas y no por el hombre.

Y entre las ajenas, las ambientales de circunstancias meteorológicas en que están trabajando.

Gran parte de los accidentes son debidos al factor humano y de aquí que ya podemos claramente compren- 
der el porqué al trabajar en seguridad debemos colaborar con todas nuestras fuerzas los técnicos y los médicos, colaboración probada y con resultados magníficos en aquellos casos en que se ha llevado a cabo.

En nuestro país se ocupan de la Seguridad una serie de organismos oficiales dependientes principalmente del Ministerio de Trabajo, entre ellos el Instituto de Medicina y Seguridad en el Trabajo, Plan nacional y otra serie de entidades privadas entre las que, a título de ejemplo, citamos: A.P.A. (Asociación para la Prevención de Accidentes, fundada por las Compañías de Seguros):

\section{- C.S.I.S. Industria Siderúrgica.}

- A.M.Y.S. Industria Hidraúlica.

- A.S.I.Q. Industria Química.

- SEOPAN Industria Construcción. Empresas de ámbito nacional.

Con todo esto he querido presentar un panorama general sobre la Seguridad para que podamos valorar la necesidad e importancia de preocuparnos por suprimir, o al menos atenuar, los accidentes y sus repercusiones; ver también cómo un profesional entra de lleno en este aspecto y, de ahí, que estimemos hay pocos cursos para Ingenieros, y más para mandos medios, encargados, vigilantes y obreros.

El Ingeniero, como antes decimos, se encuentra inmerso en su vida profesional en la cadena de producción, bien sea en la etapa de proyecto, en la de construcción o en la de explotación, y puede ser desde el lado de la Administración o de la Empresa privada.

A alguien puede que le choque el que hayamos señalado en primer lugar al Proyecto, cuando estamos hablando de Seguridad, pero la verdadera Seguridad debe arrancar del proyecto, debemos llegar a la Seguridad Integrada.
La inmensa mayoría de nuestros compañeros toma contacto con el accidente en la construcción. ¿Cuáles son sus responsabilidades en el mismo? Sin hacer una relación exhaustiva, desea obtener un producto bien hecho (calidad), por un costo mínimo (economía), en un tiempo determinado (plazo) y, para muchos, ahí termina su responsabilidad en lograr conjuntar estas tres facetas, olvidando que tan importante como ellas es el que todo ello se haga dentro de la máxima seguridad para todos los que intervienen en la producción, lo que, en definitiva, influirá y de forma muy notoria en las tres primeras: calidad, economía y plazo.

No olvidemos que cuando se trabaja en un sitio sin peligro toda la atención se vuelve sobre lo que se está haciendo, se hace más deprisa al no tener que estar pendiente de determinadas preocupaciones y, por ello, sale más barato al aumentar el rendimiento.

La intervención del Ingeniero en los temas de Seguridad no es nueva en los últimos años, puesto que siempre los ha habido con vocación por ella y otros que sin darse cuenta la tenían presente en su actuación, pero sí es nueva la institucionalización de la figura del Ingeniero de Seguridad y del Servicio Técnico de Seguridad.

Salvo casos rarísimos, su dedicación al tema de la Seguridad era parcial, esporádica, puesto que no se les había descargado de sus funciones de directores o jefes de obra o en cualquier otro nivel de la Empresa.

Con el Plan nacional se pusieron en marcha los Servicios Técnicos de Seguridad, en principio obligatorios en las obras que tenían el Servicio Médico de Empresa, y aunque aún no está bien definida esta nueva figura del Técnico de Seguridad, en la práctica se ha avanzado con cierta rapidez y ya no es raro encontrar, en las grandes Empresas Constructoras, Técnicos e Ingenieros en plena dedicación para la Seguridad.

El Técnico de Seguridad tiene que resolver muchos casos en la doble faceta de la Seguridad y la Técnica, con- 
juntándolas y colaborando con las otras personas que trabajan en Seguridad, formando equipos con ellos.

Hay que realizar proyectos de Seguridad en los que el Técnico es tan necesario, como para hacer el plan de obra.

En estos últimos años esta toma de conciencia, de que hablábamos al principio sobre la importancia de la Seguridad, ha cambiado la manera de enfocar lo que hasta ahora se hacía en este campo y se ha logrado romper barreras de incomprensión, de competencia mal entendida y alcanzar una gran sinceridad y colaboración entre todos los que en el campo de la Seguridad trabajamos, sea del lado de la Administración o de la Contrata, y dentro de ésta aunque se pertenezca a distintas Empresas.

Se ha hecho una propuesta de normalización de todas las señalizaciones referentes a Seguridad para las obras; ha colaborado en la preparación de las fichas técnicas que está adoptando el Ministerio de la Vivienda (en lo referente a la parte de Seguridad de las mismas), y mientras se hace una homologación oficial a través del Plan nacional, está tratando de homologar parte del material de Prevención y Seguridad, así como buscando la forma de lograr unificar los datos estadísticos que manejamos todos. No olvidemos que en este año habrá unas Directivas Europeas que nos obligarán a todos.

Lo que decimos no añade más responsabilidad al Jefe de Obra o al Ingeniero en su vida profesional, ya que aunque él no se dé cuenta, desde el momento de ejercer un mando la responsabilidad de hacerlo, para lograr la máxima seguridad del personal a sus ordenes, la tiene; lo que a veces le ocurre es que le faltan conocimientos para organizar bien los tajos y la marcha general de la obra desde el lado de la Seguridad.

Como hoy día se tiende a la especialización, cada vez está más claro que una misma persona, aun dentro de una profesión y una determinada técnica, no puede saberlo todo, y de ahí la especialización de unos como Ingenieros en Seguridad añadida a sus conocimientos técnicos, para que actuando como Técnico de Seguridad y dentro de los Servicios Técnicos de Seguridad de las Empresas, asesore y ayude a los demás compañeros en el proceso de producción, bien entendido que su labor siempre debe ser de ayuda y asesoramientos, pero nunca de suplantación en la línea de mando.

Como final, en esta rápida pasada por el actual planteamiento de la Seguridad podemos señalar, en orden a la prevención de accidentes, tres etapas:

1. Sensibilización respecto a la prevención de accidentes y promoción de medios de protección personal, a fin de evitar las lesiones, junto a una normalización y homologación de estos equipos.

2. Promoción de medios colectivos de Seguridad a fin de evitar no sólo las lesiones, sino incluso los accidentes, acompañándose de formación.

3. Consiste en la integración de la Seguridad en los procedimientos de ejecución. Es, no sólo evitar la lesión y el accidente, sino incluso el riesgo, siendo el paso definitivo para una labor efectiva. Es la Seguridad Integrada. Integración dentro de los propios trabajos de la Construcción, de la Seguridad como una faceta más de ellos. Hoy día hay Técnicos que ven en este sentido la ejecución de chimeneas, túneles..., como en el Puente de los Franceses en Madrid.

Mientras la Seguridad no esté automáticamente integrada con todas sus consecuencias en nuestra industria, todas las acciones que se promuevan nacerán, y nacen de hecho, con una posibilidad de eficacia limitada.

La misión del Técnico de Seguridad es difícil y delicada, no sólo debe saber, sino que debe transmitir 
sus conocimientos y convencer a los demás; para lograr esto se impone una formación a alto nivel que deben impartir los medios oficiales.

Los hombres que ya han realizado las enseñanzas técnicas básicas en sus respectivas carreras y tengan una cierta experiencia en la construcción, un año como mínimo, son los adecuados, para que si su preocupación social se lo aconseja y pide reciba la información de prevención que haga de ellos hombres completos de la Seguridad.

En estos estudios, además de que las Técnicas de Prevención no retrasen o entorpezcan la ejecución material de la obra, no olvidemos nunca que la Seguridad debe ser el denominador común de todo trabajo bien hecho.

Hemos tratado del Servicio Técnico de Seguridad y del Técnico de Seguridad. A algunos Jefes de Obra les afecta oir esto y reaccionan de dos formas:
- Que lata, ya me van a venir incordiando, poniéndome trabas, dándome órdenes... No hay duda, no han entendido nada, ni de Seguridad Integrada ni de Servicios Técnicos o Técnico de Seguridad.

- O por el contrario, iqué bien!, que me digan qué hago y la responsabilidad será de ellos, me libero de ella. Craso error, igual que el anterior.

La Seguridad es misión del Jefe de Obra.

El Jefe de Obra, al aceptar la jefatura ha asumido íntegramente una serie de responsabilidades y entre ellas, en lugar importante, la Seguridad. Responde ante sus compañeros y superiores del resultado de su gestión, y en ésta entra el acabar la obra sin accidentes.

Los Servicios Técnicos le ayudarán, le asesorarán, pero no le sustituirán en su responsabilidad, y él debe contribuir a integrar la Seguridad en la obra.

\section{publicación del ICCET/CSIC}

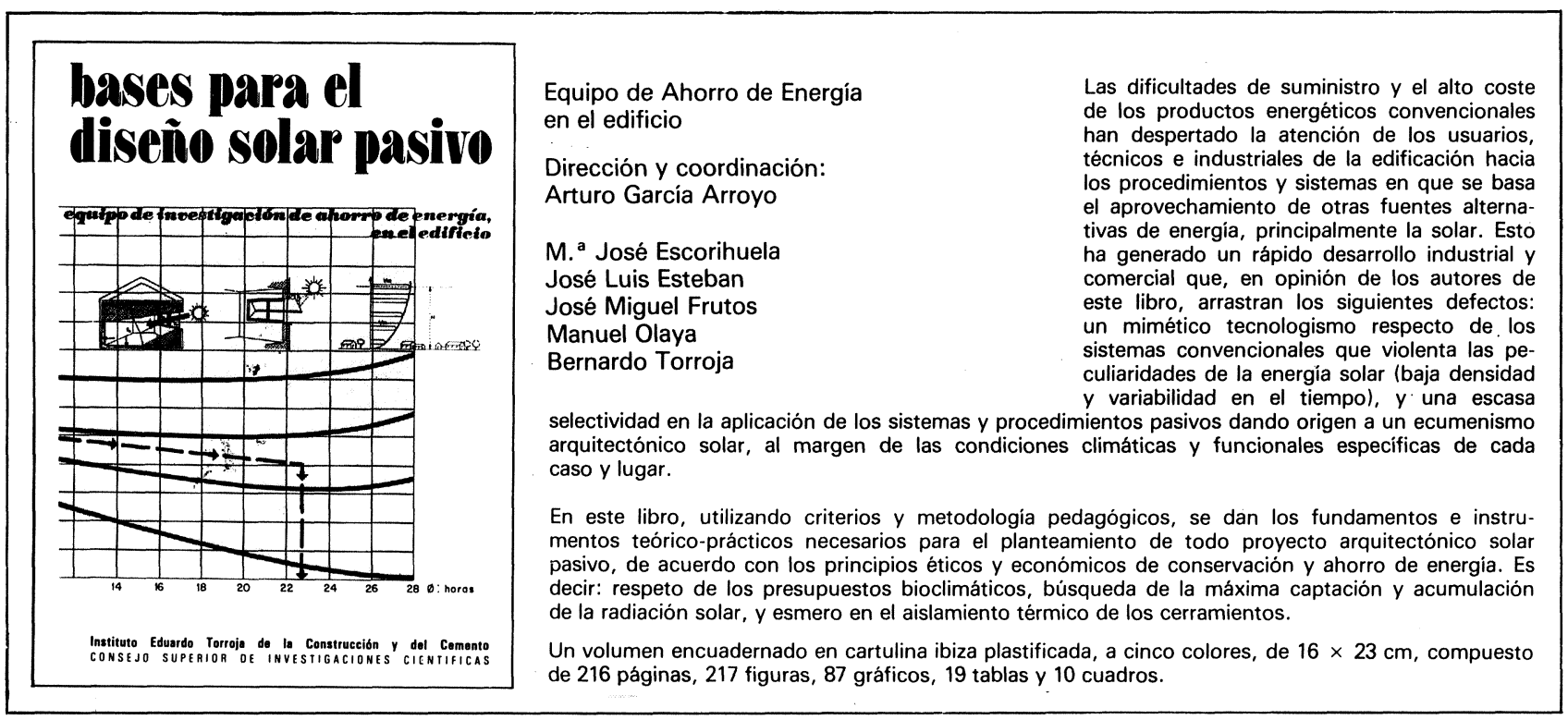

